\title{
Computation of generalized inverses of periodic systems
}

\author{
A. Varga
}

\begin{abstract}
We address the numerically reliable computation of generalized inverses of periodic systems. The underlying inverses are defined via the corresponding lifted representations. Structure preserving reduction of the associated system pencil to a special Kronecker-like form is the main computational ingredient for the proposed approach. This form can be computed by employing exclusively orthogonal transformations. For the computational algorithm of the generalized inverse, the backward numerical stability can be proved.
\end{abstract}

\section{INTRODUCTION}

Inverse systems have many important applications in areas such as control theory, filtering and coding theory. The computation of system inverses for standard linear time-invariant systems is essentially equivalent to compute generalized inverses of the associated transfer-function matrices. We formulate an equivalent problem for the inversion of discrete-time periodic systems in terms of the transferfunction matrix of the associated lifted representation.

For square and invertible periodic systems, the computation of inverses can be done by explicit formulas. For non-square systems, explicit formulas can be employed only in the full-rank case to determine left or right inverses, provided the system feedthrough matrices $D_{k}$ have full ranks. However, these direct formulas do not allow to arbitrarily choose the spurious poles which appear in the computed left or right inverses. In the more general case of periodic systems with transfer-function matrices of arbitrary rank, no explicit formulas can be used.

The inversion problem for periodic systems has been considered by several authors [1], [2], by deriving conditions for left/right invertibility. A constructive approach to compute left/right inverses has been developed in [2], based on a recursive procedure proposed in [3], which is similar to Silverman's structure algorithm [4]. The main advantage of this approach is that the resulting inverse system is already in a state-space form. Note that the existence of equivalent procedures to determine inverses using liftingbased approaches is mentioned in [2] as still an open problem.

In this paper we address the computation of periodic week generalized inverses (known also as the (1,2)-inverses) starting from the lifted-formulation of the problem. This formulation has the main theoretical advantage that the system inversion concepts related to compute various generalized inverses (see for example [5]) can be easily ex-

A. Varga is with the German Aerospace Center, DLR - Oberpfaffenhofen, Institute of Robotics and Mechatronics, D-82234 Wessling, Germany. E-mail: andras.varga@dlr.de pressed in terms of the transfer-function matrix (TFM) of the associated lifted representation. A straightforward but numerically naive approach to compute periodic inverses would be to apply first the method of [5] to the lifted representation and then to compute a minimal realization of the resulting TFM of the inverse to obtain a statespace periodic system description. This simple approach has several disadvantages. Firstly, the resulting TFM can be improper and, at present, there exists no satisfactory algorithm to convert a general descriptor system representation into a periodic one. Even in the case when the resulting inverse is proper (thus, the realization algorithm of [6] can be employed), the use of lifted representations with large order sparse matrices represent a computational challenge for large periods or state dimensions. Besides this, because this method completely ignores the structure of the lifted system matrices, there is no guarantee for a satisfactory numerical performance. For example, the strong numerical stability of computations is not ensured even when applying numerically stable algorithms in all computational steps.

In what follows, we propose a numerically reliable procedure to construct generalized inverses which exploits and preserves the sparse structure of the lifted system matrices and generates directly a state-space representation of the inverse. This procedure relies on structure preserving orthogonal transformations to compute particular Kronecker-like form of the lifted system pencil. It can be shown that the computational algorithm of the inverse is backward numerically stable and has a low computational complexity which is linear in the period and cubic in the maximal problem dimensions. The resulting inverse is minimal, provided the initial description of the system (1) is minimal. Thus, the computational algorithm is completely satisfactory according to the criteria we formulated in [7]. In combination with stabilization algorithms for periodic systems, the proposed procedure can also cope with constraints related to the stability of the inverse. For example, stable inverses of minimum-phase systems can be easily constructed. A parameterization of all $(1,2)$-inverses in terms of left and right annihilators can be employed to address the computation of least order inverses.

Notation. For an $N$-periodic matrix $X_{i}$ we use systematically the script notation

$$
\mathcal{X}_{k}:=\operatorname{diag}\left(X_{k}, X_{k+1}, \ldots, X_{k+N-1}\right),
$$

to denote the block-diagonal matrix $\mathcal{X}_{k}$ associated to the cyclic matrix sequence $X_{i}, i=k, \ldots, k+N-1$ starting at time moment $k$. 


\section{Preliminaries}

We consider periodic time-varying descriptor systems of the form

$$
\begin{aligned}
E_{k} x(k+1) & =A_{k} x(k)+B_{k} u(k) \\
y(k) & =C_{k} x(k)+D_{k} u(k)
\end{aligned}
$$

where the matrices $E_{k} \in \mathrm{R}^{\mu_{k} \times n_{k+1}}, A_{k} \in \mathrm{R}^{\mu_{k} \times n_{k}}$, $B_{k} \in \mathrm{R}^{\mu_{k} \times m_{k}}, C_{k} \in \mathrm{R}^{p_{k} \times n_{k}}, D_{k} \in \mathrm{R}^{p_{k} \times m_{k}}$ are periodic with period $N \geq 1$, and the dimensions fulfil the condition $\nu=\sum_{k=1}^{N} \mu_{k}=\sum_{k=1}^{N} n_{k}$. By using the script notation, the periodic system (1) will be alternatively denoted by the quintuple $\left(\mathcal{E}_{k}, \mathcal{A}_{k}, \mathcal{B}_{k}, \mathcal{C}_{k}, \mathcal{D}_{k}\right)$.

To define the inverse of the periodic system (1), we define first the TFM corresponding to the associated stacked lifted representation of [8], which uses the input-state-output behavior of the system over time intervals of length $N$, rather then 1. For a given sampling time $k$, the corresponding $M$ dimensional input vector, $P$-dimensional output vector and $\nu$-dimensional state vector are

$$
\begin{aligned}
& u_{k}^{S}(h)=\left[u^{T}(k+h N) \cdots u^{T}(k+h N+N-1)\right]^{T}, \\
& y_{k}^{S}(h)=\left[y^{T}(k+h N) \cdots y^{T}(k+h N+N-1)\right]^{T}, \\
& x_{k}^{S}(h)=\left[x^{T}(k+h N) \cdots x^{T}(k+h N+N-1)\right]^{T} .
\end{aligned}
$$

where $M=\sum_{k=1}^{N} m_{k}$ and $P=\sum_{k=1}^{N} p_{k}$. The corresponding lifted system can be represented by a time-invariant descriptor system of the form

$$
\begin{aligned}
L_{k} x_{k}^{S}(h+1) & =F_{k} x_{k}^{S}(h)+G_{k} u_{k}^{S}(h) \\
y_{k}^{S}(h) & =H_{k} x_{k}^{S}(h)+J_{k} u_{k}^{S}(h)
\end{aligned}
$$

where $G_{k}=\mathcal{B}_{k}, H_{k}=\mathcal{C}_{k}, J_{k}=\mathcal{D}_{k}$, and

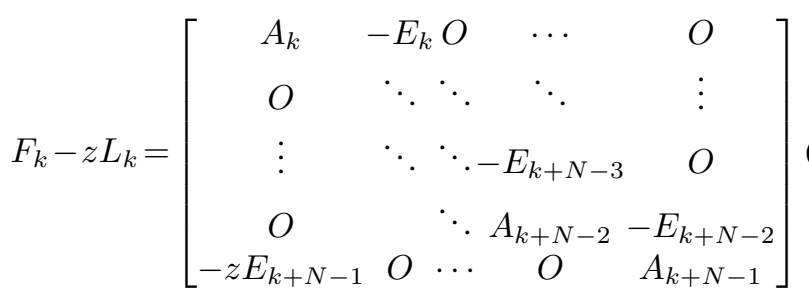

Assuming this square pencil is regular (i.e., $\operatorname{det}\left(F_{k}-z L_{k}\right) \not \equiv$ 0 ), the TFM of the lifted system is

$$
W_{k}(z)=H_{k}\left(z L_{k}-F_{k}\right)^{-1} G_{k}+J_{k}
$$

and the associated system pencil is defined as

$$
P_{k}(z)=\left[\begin{array}{cc}
F_{k}-z L_{k} & G_{k} \\
H_{k} & J_{k}
\end{array}\right],
$$

which both depend on the sampling time $k$. Obviously $W_{k+N}(z)=W_{k}(z)$ and the TFMs at two successive values of $k$ are related by the following relation [9]

$$
W_{k+1}(z)=\left[\begin{array}{cc}
0 & I_{P-p_{k}} \\
z I_{p_{k}} & 0
\end{array}\right] W_{k}(z)\left[\begin{array}{cc}
0 & z^{-1} I_{m_{k}} \\
I_{M-m_{k}} & 0
\end{array}\right]
$$

To define poles and zeros of the periodic system (1), we need to assume the minimality of the system (1) and thus of the lifted realization (2). This is equivalent to the notion of reachability and observability at finite and infinite eigenvalues of the pencil (5), as introduced in [10]. If we assume that the system (1) is minimal in that sense (this implies time-varying state dimensions and rectangular descriptor matrices) then we have the following definitions of poles, zeros and minimal indices of the TFM (4) in terms of the system matrix (5).

Definition 1: The poles of the transfer function $W_{k}(z)$ of the minimal periodic system (1) are the zeros of the associated pole pencil $F_{k}-z L_{k}$ defined in (3).

Definition 2: The transmission zeros of the transfer function $W_{k}(z)$ of the minimal periodic system (1) are the invariant zeros of the associated stacked system pencil (5).

Definition 3: The left and right minimal indices of the transfer function $W_{k}(z)$ of the minimal periodic system (1) are those of the associated stacked system pencil (5).

It follows from (6) that poles and zeros of the TFMs for different sampling times, can only differ at $z=0$ and $z=\infty$.

Consider now the permuted system matrix $\widetilde{P}_{k}(z)=$ $\Pi_{1} P_{k}(z) \Pi_{2}$

$$
\widetilde{P}_{k}(z)=\left[\begin{array}{ccccc}
S_{k} & -T_{k} & O & \cdots & O \\
O & S_{k+1} & -T_{k+1} & \cdots & O \\
\vdots & \ddots & \ddots & \ddots & \vdots \\
O & & & S_{k+N-2} & -T_{k+N-2} \\
-z T_{k+N-1} & O & \cdots & O & S_{k+N-1}
\end{array}\right]
$$

where for $\mathrm{i}=k, k+1, \ldots, k+N-1$

$$
S_{i}=\left[\begin{array}{cc}
A_{i} & B_{i} \\
C_{i} & D_{i}
\end{array}\right], \quad T_{i}=\left[\begin{array}{cc}
E_{i} & O \\
O & O
\end{array}\right]
$$

The above definitions of zeros and minimal indices can be evidently given also in terms of this permuted pencil. By suitably redefining the matrices of the periodic pair $\left(S_{k}, T_{k}\right)$, we can define for non-minimal systems special types of zeros which, according to [8], characterize the reachability and observability properties.

Definition 4: The input decoupling zeros of the periodic system (1) are the invariant zeros of the pencil $\widetilde{P}_{k}(z)$ in (7) for

$$
S_{i}:=\left[\begin{array}{ll}
A_{i} & B_{i}
\end{array}\right], \quad T_{i}:=\left[\begin{array}{cc}
E_{i} & O
\end{array}\right]
$$

Definition 5: The output decoupling zeros of the periodic system (1) are the invariant zeros of the pencil $\widetilde{P}_{k}(z)$ in (7) for

$$
S_{i}:=\left[\begin{array}{c}
A_{i} \\
C_{i}
\end{array}\right], \quad T_{i}:=\left[\begin{array}{c}
E_{i} \\
O
\end{array}\right]
$$

By extending the definitions of [8], the system (1) is reachable if it has no input decoupling zeros, and is observable if it has no output decoupling zeros. Note that all above definitions are valid for a specified time moment $k$. We say that the system (1) is completely reachable if it is reachable for all $k$, and completely observable if it is observable for all $k$. The periodic system (1) is minimal if it is completely reachable and completely observable. Obviously, these definitions generalize those for standard periodic systems (i.e., with $E_{k}=I_{n_{k+1}}$ ) [8]. 


\section{Computation of $(1,2)$-Inverses}

We can define the (1,2)-inverses of the periodic system (1) in terms of the $(1,2)$-inverses of the associated $P \times M$ rational TFM $W_{k}(z)$. Specifically, a $(1,2)$-inverse $X_{k}(z)$ of $W_{k}(z)$ must satisfy the first and second so-called MoorePenrose relations (see e.g., [12]):

$$
\begin{aligned}
& \text { 1) } W_{k}(z) X_{k}(z) W_{k}(z)=W_{k}(z) \\
& X_{k}(z) W_{k}(z) X_{k}(z)=X_{k}(z)
\end{aligned}
$$

We denote a $(1,2)$-inverse (or week generalized inverse) $X_{k}(z)$ satisfying the above conditions as $W_{k}^{+}(z)$. Note that, the left and right inverses, frequently used in the control literature, are particular (1,2)-inverses of systems with full column rank or full row rank TFMs, respectively.

The computation of $(1,2)$-inverses of $W_{k}(z)$ can be done using the following straightforward formula

$$
W_{k}^{+}(z)=\left[\begin{array}{ll}
0 & I_{M}
\end{array}\right] P_{k}^{+}(z)\left[\begin{array}{c}
0 \\
I_{P}
\end{array}\right],
$$

where $P_{k}^{+}(z)$ is an $(1,2)$-inverse of $P_{k}(z)$. If we use the permuted system matrix $\widetilde{P}_{k}(z)=\Pi_{1} P_{k}(z) \Pi_{2}$ in (7), then (9) can be equivalently written as

$$
W_{k}^{+}(z)=\widetilde{\mathcal{C}} \widetilde{P}_{k}^{+}(z) \widetilde{\mathcal{B}}
$$

where $\widetilde{C}_{k}=\left[\begin{array}{ll}0 & I_{m_{k}}\end{array}\right]$ and $\widetilde{B}_{k}=\left[\begin{array}{c}0 \\ I_{p_{k}}\end{array}\right]$. In the case of a locally square system (i.e., $p_{k}=m_{k}$ ), an explicit inverse can be immediately defined as a periodic descriptor system

$$
\begin{aligned}
T_{k} \widetilde{x}(k+1) & =S_{k} \widetilde{x}(k)-\widetilde{B}_{k} \widetilde{u}(k) \\
\widetilde{y}(k) & =\widetilde{C}_{k} \widetilde{x}(k)
\end{aligned}
$$

Provided $D_{k}$ is invertible for $k=1, \ldots, N$, we can also express the above inverse in the more familiar form

$$
\begin{aligned}
E_{k} \bar{x}(k+1) & =\left(A_{k}-B_{k} D_{k}^{-1} C_{k}\right) \bar{x}(k)-B_{k} D_{k}^{-1} \widetilde{u}(k) \\
\widetilde{y}(k) & =D_{k}^{-1} C_{k} \bar{x}(k)+D_{k}^{-1} \widetilde{u}(k)
\end{aligned}
$$

We will need the following result to compute $(1,2)$ inverses of non-square systems (see e.g., [12]):

Lemma 1: Let $R(z)$ be a rational matrix of rank $\ell$ and let $\Pi_{l}$ and $\Pi_{r}$ permutation matrices such that

$$
\Pi_{l} R(z) \Pi_{r}=\left[\begin{array}{ll}
R_{11}(z) & R_{12}(z) \\
R_{21}(z) & R_{22}(z)
\end{array}\right]
$$

where $\operatorname{rank} R_{11}(z)=\ell$. Then, an (1,2)-inverse of $R(z)$ is

$$
R^{+}(z)=\Pi_{r}\left[\begin{array}{cc}
R_{11}^{-1}(z) & 0 \\
0 & 0
\end{array}\right] \Pi_{l} .
$$

According to this lemma, the computation of $\widetilde{P}_{k}^{+}(z)$ amounts to isolate first a maximal rank submatrix of $P_{k}(z)$ and then to apply the above formula. This operation can be done by reducing $\widetilde{P}_{k}(z)$ to an appropriate Kronecker-like form from which a maximal rank regular sub-pencil can be easily separated. Let $Q_{k}$ and $Z_{k}$ be orthogonal $N$-periodic matrices determined using the algorithm proposed in [11] to reduce the $N$-periodic pair $\left(S_{k}, T_{k}\right)$ to the Kronecker-like form $\left(\bar{S}_{k}, \bar{T}_{k}\right):=\left(Q_{k} S_{k} Z_{k}, Q_{k} T_{k} Z_{k+1}\right)$, where

$$
\begin{gathered}
\bar{S}_{k}=\left[\begin{array}{c|c}
\bar{S}_{k}^{11} & \bar{S}_{k}^{12} \\
\hline 0 & \bar{S}_{k}^{22}
\end{array}\right]=\left[\begin{array}{c|ccc}
B_{k}^{r} & A_{k}^{r} & * & * \\
O & O & A_{k}^{r e g} & * \\
O & O & O & A_{k}^{l} \\
\hline O & O & O & C_{k}^{l}
\end{array}\right] \\
\bar{T}_{k}=\left[\begin{array}{c|c|c}
O & \bar{T}_{k}^{12} \\
\hline O & O
\end{array}\right]=\left[\begin{array}{c|ccc}
O & E_{k}^{r} & * & * \\
O & O & E_{k}^{r e g} & * \\
O & O & O & E_{k}^{l} \\
\hline O & O & O & O
\end{array}\right]
\end{gathered}
$$

where: (a) $\mathcal{E}_{k}^{r}$ is invertible and the periodic system $\left(\mathcal{E}_{k}^{r}, \mathcal{A}_{k}^{r}, \mathcal{B}_{k}^{r}, *, *\right)$ is completely reachable; (b) $\mathcal{E}_{k}^{l}$ is invertible and the periodic system $\left(\mathcal{E}_{k}^{l}, \mathcal{A}_{l}^{r}, *, \mathcal{C}_{k}^{l}, *\right)$ is completely observable; (c) the pole pencil of the form (3) corresponding to the pair $\left(\mathcal{E}_{k}^{\text {reg }}, \mathcal{A}_{k}^{\text {reg }}\right)$ is regular. Note that the triples $\left(\mathcal{E}_{k}^{r}, \mathcal{A}_{k}^{r}, \mathcal{B}_{k}^{r}\right)$ and $\left(\mathcal{E}_{k}^{l}, \mathcal{A}_{k}^{l}, \mathcal{C}_{k}^{l}\right)$ specify the right and left Kronecker structures of $P_{k}(z)$, respectively, while the pair $\left(\mathcal{E}_{k}^{r e g}, \mathcal{A}_{k}^{r e g}\right)$ specifies the finite and infinite zero structure of $P_{k}(z)$. Consider partitions compatible with (13) for $Q_{k} \widetilde{B}_{k}$ and $C_{k} Z_{k}$

$$
Q_{k} \widetilde{B}_{k}=\left[\frac{\bar{B}_{k}^{1}}{\bar{B}_{k}^{2}}\right], \quad \widetilde{C}_{k} Z_{k}=\left[\bar{C}_{k}^{1} \mid \bar{C}_{k}^{2}\right]
$$

With appropriate permutation matrices $\Pi_{l}$ and $\Pi_{r}$ it is possible to rearrange the transformed pencil $\mathcal{Q}_{k} \widetilde{P}_{k}(z) \mathcal{Z}_{k}$ such that

$$
\bar{P}_{k}(z):=\Pi_{l} \mathcal{Q}_{k} \widetilde{P}_{k}(z) \mathcal{Z}_{k} \Pi_{r}=\left[\begin{array}{c|c}
\bar{P}_{k}^{11}(z) & \bar{P}_{k}^{12} \\
\hline \bar{P}_{k}^{21} & O
\end{array}\right]
$$

where $\bar{P}_{k}^{11}(z)$ is the square and invertible pencil

$$
\bar{P}_{k}^{11}(z)=\left[\begin{array}{ccccc}
\bar{S}_{k}^{12} & -\bar{T}_{k}^{12} & O & \cdots & O \\
O & \bar{S}_{k+1}^{12} & -\bar{T}_{k+1}^{12} & \cdots & O \\
\vdots & \ddots & \ddots & \ddots & \vdots \\
O & & & \bar{S}_{k+N-2}^{12} & -\bar{T}_{k+N-2}^{12} \\
-z \bar{T}_{k+N-1}^{12} & O & \cdots & O & \bar{S}_{k+N-1}^{12}
\end{array}\right]
$$

and $\bar{P}_{k}^{12}=\overline{\mathcal{S}}_{k}^{11}, \bar{P}_{k}^{21}=\overline{\mathcal{S}}_{k}^{22}$. We define and partition analogously

$$
\overline{\mathcal{B}}_{k}=\Pi_{l} \mathcal{Q}_{k} \widetilde{\mathcal{B}}_{k}=\left[\frac{\overline{\mathcal{B}}_{k}^{1}}{\overline{\mathcal{B}}_{k}^{2}}\right], \quad \overline{\mathcal{C}}_{k}=\widetilde{\mathcal{C}}_{k} \mathcal{Z}_{k} \Pi_{r}=\left[\overline{\mathcal{C}}_{k}^{2} \mid \overline{\mathcal{C}}_{k}^{1}\right]
$$

and we have immediately that

$$
W_{k}^{+}(z)=\overline{\mathcal{C}}_{k} \bar{P}_{k}^{+}(z) \overline{\mathcal{B}}_{k}
$$

The following result is straightforward.

Lemma 2:

$$
\operatorname{rank} \bar{P}_{k}(z)=\operatorname{rank} \bar{P}_{k}^{11}(z)
$$

By applying now Lemma 1, we obtain

$$
W_{k}^{+}(z)=\overline{\mathcal{C}}_{k}\left[\begin{array}{c|c}
{\left[\bar{P}_{k}^{11}(z)\right]^{-1}} & O \\
\hline O & O
\end{array}\right] \overline{\mathcal{B}}_{k}=\overline{\mathcal{C}}_{k}^{2}\left[\bar{P}_{k}^{11}(z)\right]^{-1} \overline{\mathcal{B}}_{k}^{1}
$$


It follows that a realization of this (1,2)-inverse of $W_{k}(z)$ is defined by the periodic descriptor system

$$
\begin{aligned}
\bar{T}_{k}^{12} \bar{x}(k+1) & =\bar{S}_{k}^{12} \bar{x}(k)-\bar{B}_{k}^{1} \bar{u}(k) \\
\bar{y}(k) & =\bar{C}_{k}^{2} \bar{x}(k)
\end{aligned}
$$

\section{PROPERTIES OF $(1,2)$-INVERSES}

By exploiting the fine structure of the pair $\left(\bar{S}_{k}, \bar{T}_{k}\right)$, it is possible to bring the pencil $\bar{P}_{k}(z)$ in (15) using appropriate permutation matrices $\Pi_{3}$ and $\Pi_{4}$ in the form

$$
\Pi_{3} \bar{P}_{k}(z) \Pi_{4}=\left[\begin{array}{ccc|c}
P_{k}^{r}(z) & * & * & \mathcal{B}_{k}^{r} \\
\mathcal{O} & P_{k}^{r e g}(z) & * & \mathcal{O} \\
\mathcal{O} & \mathcal{O} & P_{k}^{l}(z) & \mathcal{O} \\
\hline \mathcal{O} & \mathcal{O} & \mathcal{C}_{k}^{l} & \mathcal{O}
\end{array}\right]
$$

where, for $x=r$, reg, $l$,

$$
P_{k}^{x}(z)=\left[\begin{array}{ccccc}
A_{k}^{x} & -E_{k}^{x} & O & \cdots & O \\
O & A_{k+1}^{x} & -E_{k+1}^{x} & \cdots & O \\
\vdots & \ddots & \ddots & \ddots & \vdots \\
O & & & A_{k+N-2}^{x}-E_{k+N-2}^{x} \\
-z E_{k+N-1}^{x} & O & \cdots & O & E_{k+N-1}^{x}
\end{array}\right]
$$

We have the following straightforward result.

Proposition 1: The transmission zeros of the minimal periodic system (1) are the zeros of the subpencil $P_{k}^{r e g}(z)$.

Proof: From the construction of the Kronecker-like form of the pair $\left(S_{k}, T_{k}\right)$, the subpencils $\left[\mathcal{B}_{k}^{r} P_{k}^{r}(z)\right]$ and $\left[\begin{array}{c}P_{k}^{l}(z) \\ \mathcal{C}_{k}^{l}\end{array}\right]$ have, respectively, full row and full column ranks for all $z$ (finite and infinite). Therefore, the zeros of regular subpencil $P_{k}^{r e g}(z)$ represent the transmission zeros of the periodic system (1) (where the system pencil (5) looses its normal rank).

Note that in the case of a non-minimal system, the zeros of $P_{k}^{r e g}(z)$ contains additionally the input and output decoupling zeros of the system.

We can easily prove the following well known fact.

Proposition 2: The poles of the inverse system (16) contains the transmission zeros of the original system.

Proof: We assume the original system is minimal. Since the pole pencil of the inverse is the permuted leading diagonal block of (17), we have merely to show that the system zeros, contained in $P_{k}^{r e g}(z)$, are completely reachable and observable for the corresponding input and output matrices $\widehat{\mathcal{B}}_{k}^{1}$ and $\widehat{\mathcal{C}}_{k}^{2}$, where

$$
\begin{aligned}
& \Pi_{3} \overline{\mathcal{B}}_{k}=\left[\begin{array}{c}
\widehat{\mathcal{B}}_{k}^{1} \\
\hline \overline{\mathcal{B}}_{k}^{2}
\end{array}\right]=\left[\begin{array}{l}
\widehat{\mathcal{B}}_{k}^{r} \\
\widehat{\mathcal{B}}_{k}^{r e g} \\
\widehat{\mathcal{B}}_{k}^{l} \\
\hline \overline{\mathcal{B}}_{k}^{2}
\end{array}\right]
\end{aligned}
$$

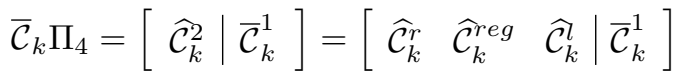

To check observability, consider the extended pencil

$$
P_{k}^{e}(z)=\left[\begin{array}{cc}
F_{k}-z L_{k} & G_{k} \\
H_{k} & J_{k} \\
\hdashline 0 & I_{M}
\end{array}\right]
$$

and transform it as follows

$$
\begin{gathered}
{\left[\begin{array}{cc}
\Pi_{3} \Pi_{l} \mathcal{Q}_{k} \Pi_{1} & \mathcal{O} \\
O & I_{M}
\end{array}\right] P_{k}^{e}(z) \Pi_{2} \mathcal{Z}_{k} \Pi_{r} \Pi_{4}=} \\
{\left[\begin{array}{ccc|c}
P_{k}^{r}(z) & * & * & \mathcal{B}_{k}^{r} \\
\mathcal{O} & P_{k}^{r e g}(z) & * & \mathcal{O} \\
\mathcal{O} & \mathcal{O} & P_{k}^{l}(z) & \mathcal{O} \\
\hline \mathcal{O} & \mathcal{O} & \mathcal{\mathcal { C }} & \mathcal{O} \\
\hdashline \hat{\mathcal{C}}_{k}^{r} & \mathcal{\mathcal { C }}_{k}^{r e g} & \mathcal{\mathcal { C }}_{k}^{l} & \overline{\mathcal{C}}_{k}^{1-}
\end{array}\right]}
\end{gathered}
$$

Since $P_{k}^{e}(z)$ has full column rank, it follows that

$$
\left[\begin{array}{cc}
P_{k}^{r}(z) & * \\
\mathcal{O} & P_{k}^{r e g}(z) \\
\hdashline \widehat{\mathcal{C}}_{k}^{r} & \widehat{\mathcal{C}}_{k}^{r e g}
\end{array}\right]
$$

has full column rank as well. Thus, the zeros of $P_{k}^{r e g}(z)$ are observable. The reachability of the zeros can be shown similarly.

From the properties of submatrices in the Kroneckerlike form (13) and (14) we can check immediately the existence of right (left) inverses and characterize some of their properties.

Proposition 3: The original system (1) is right (left) invertible if the pair $\left(\bar{S}_{k}, \bar{T}_{k}\right)$ has no right (left) Kronecker structure.

The following result is practically a corollary of Proposition 2.

Proposition 4: Any right (left) inverse of the form (16) is completely observable (reachable) provided the original system (1) is completely observable (reachable).

Proof: In the case of a right inverse, consider once again the extended pencil (18). Since the original system is completely observable, this pencil has full column rank for all $k$. Consider the transformed extended pencil

$$
\left[\begin{array}{cc}
\Pi_{l} \mathcal{Q}_{k} \Pi_{1} & O \\
O & I_{M}
\end{array}\right] P_{k}^{e}(z) \Pi_{2} \mathcal{Z}_{k} \Pi_{r}=\left[\begin{array}{c|c}
\bar{P}_{k}^{11}(z) & \bar{P}_{k}^{12} \\
\hdashline \underline{\mathcal{C}}_{k}^{-2} & \overline{\mathcal{C}}_{k}^{1--}
\end{array}\right]
$$

which has also full column rank. It follows that $\left[\begin{array}{c}\bar{P}_{k}^{11}(z) \\ \overline{\mathcal{C}}_{k}^{2}\end{array}\right]$ has full column rank too and thus the inverse system is completely observable. The proof for a left inverse is completely analogous.

Note that in general, the resulting right (left) inverse can be unreachable (unobservable) for a special choice of the transformation matrices and this feature is the key to determine least order inverses (see Section VI-C).

\section{Computation of Stable $(1,2)$-InVerses}

If the periodic system (1) is minimum-phase (i.e., has no infinite zeros and all finite zeros are stable), we are often interested to determine a stable $(1,2)$-inverse of it. From the results of previous section, it follows that the poles of the inverse always contain the system zeros, and therefore this part of poles is fixed. The rest of poles is formed from the reunion of zeros of the subpencils $P_{k}^{r}(z)$ and $P_{k}^{l}(z)$, and, as we will see, is freely assignable. These poles are sometimes 
called the "spurious poles", because they arise depending on the method used to determine the $(1,2)$-inverse.

Consider transformation matrices $U_{k}$ and $V_{k}$ of the form

$$
\begin{gathered}
U_{k}=\left[\begin{array}{ccc|c}
I & 0 & 0 & K_{k}^{1} \\
0 & I & 0 & K_{k}^{2} \\
0 & 0 & I & K_{k}^{3} \\
\hline 0 & 0 & 0 & I
\end{array}\right]:=\left[\begin{array}{c|c}
I & K_{k} \\
\hline 0 & I
\end{array}\right] \\
V_{k}=\left[\begin{array}{c|ccc}
I & F_{k}^{1} & F_{k}^{2} & F_{k}^{3} \\
\hline 0 & I & 0 & 0 \\
0 & 0 & I & 0 \\
0 & 0 & 0 & I
\end{array}\right]:=\left[\begin{array}{c|c}
I & F_{k} \\
\hline 0 & I
\end{array}\right]
\end{gathered}
$$

where the identity matrices have dimensions compatible with the block row structure of the reduced matrix $\bar{S}_{k}$ in (13). Then, the transformed pair $\left(\widehat{S}_{k}, \widehat{T}_{k}\right):=$ $\left(U_{k} \bar{S}_{k} V_{k}, U_{k} \bar{T}_{k} V_{k+1}\right)$ is given by

$$
\begin{gathered}
\widehat{S}_{k}=\left[\begin{array}{c|c}
\bar{S}_{k}^{11} \mid \widehat{S}_{k}^{12} \\
\hline 0 & \bar{S}_{k}^{22}
\end{array}\right]=\left[\begin{array}{c|ccc}
B_{k}^{r} & A_{k}^{r}+B_{k}^{r} F_{k}^{1} & * & * \\
O & O & A_{k}^{r e g} & * \\
O & O & O & A_{k}^{l}+K_{k}^{3} C_{k}^{l} \\
\hline O & & O & C_{k}^{l}
\end{array}\right] \\
\widehat{T}_{k}=\left[\begin{array}{c|c|c|c}
O & \widehat{T}_{k}^{12} \\
\hline O & O
\end{array}\right]=\left[\begin{array}{c|ccc}
O & E_{k}^{r} & * & * \\
O & O & E_{k}^{r e g} & * \\
O & O & O & E_{k}^{l} \\
\hline O & O & O & O
\end{array}\right]
\end{gathered}
$$

where $\widehat{S}_{k}^{12}=\bar{S}_{k}^{12}+\bar{S}_{k}^{11} F_{k}+K_{k} \bar{S}_{k}^{22}$. The inverse periodic system is defined by

$$
\begin{aligned}
\widehat{T}_{k}^{12} \widehat{x}(k+1) & =\widehat{S}_{k}^{12} \widehat{x}(k)-\widehat{B}_{k}^{1} \widehat{u}(k) \\
\widehat{y}(k) & =\widehat{C}_{k}^{2} \widehat{x}(k)
\end{aligned}
$$

where

$$
\begin{gathered}
U_{k} Q_{k} \widetilde{B}_{k}=\left[\frac{\widehat{B}_{k}^{1}}{\widehat{B}_{k}^{2}}\right]:=\left[\frac{\bar{B}_{k}^{1}+K_{k} \bar{B}_{k}^{2}}{\bar{B}_{k}^{2}}\right] \\
\widetilde{C}_{k} Z_{k} V_{k}=\left[\widehat{C}_{k}^{1} \mid \widehat{C}_{k}^{2}\right]:=\left[\bar{C}_{k}^{1} \mid \bar{C}_{k}^{2}+\bar{C}_{k}^{1} F_{k}\right]
\end{gathered}
$$

To obtain inverses with the spurious poles lying in a "good" domain $\mathbb{C}_{g}$ of the complex plane (e.g., stable domain), we can solve two periodic pole assignment problems. For the transformed pair $\left(\widehat{S}_{k}, \widehat{T}_{k}\right)$ the set of spurious poles of the inverse is formed from the reunion of the eigenvalues of the matrices $\left(E_{N}^{r}\right)^{-1}\left(A_{N}^{r}+B_{N}^{r} F_{N}^{1}\right) \cdots\left(E_{1}^{r}\right)^{-1}\left(A_{1}^{r}+\right.$ $\left.B_{1}^{r} F_{1}^{1}\right)$ and $\left(E_{N}^{l}\right)^{-1}\left(A_{N}^{l}+K_{N}^{3} C_{N}^{l}\right) \cdots\left(E_{1}^{l}\right)^{-1}\left(A_{1}^{l}+\right.$ $\left.K_{1}^{3} C_{1}^{l}\right)$. Thus, by choosing appropriate $F_{k}^{1}$ and $K_{k}^{3}$ all non-zero spurious poles can be moved to arbitrary locations in $\mathbb{C}_{g}$. This is guaranteed by the reachability and observability of the periodic systems $\left(\mathcal{E}_{k}^{r}, \mathcal{A}_{k}^{r}, \mathcal{B}_{k}^{r}, *, *\right)$ and $\left(\mathcal{E}_{k}^{l}, \mathcal{A}_{k}^{l}, *, \mathcal{C}_{k}^{l}, *\right)$, respectively (see Section III).

\section{Some Open Computational Problems}

\section{A. Computation of delay inverses}

The structure at infinity of the computed inverse provides full information on the minimal number of delays necessary at each output of the inverse to achieve a proper (or causal) input-output behavior. In fact, this is the inversion problem considered in [2], where the structure algorithm of [3] is employed to determine the structure at infinity and to construct explicitly a delay inverse. In the case of our approach, the information on the infinite zeros can be obtained by examining the "fine" structure of the periodic pair $\left(A_{k}^{r e g}, E_{k}^{r e g}\right)$ resulting from the computational algorithm of [11]. The matrices of this pair are obtained in the form

$$
A_{k}^{r e g}=\left[\begin{array}{cc}
A_{k}^{\infty} & A_{k}^{\infty f} \\
O & A_{k}^{f}
\end{array}\right], \quad E_{k}^{r e g}=\left[\begin{array}{cc}
E_{k}^{\infty} & E_{k}^{\infty f} \\
O & E_{k}^{f}
\end{array}\right]
$$

where the pair $\left(A_{k}^{\infty}, E_{k}^{\infty}\right)$, with $A_{k}^{\infty}$ invertible, contains the zeros structure at infinity (i.e., the infinite poles of the inverse), and the pair $\left(A_{k}^{f}, E_{k}^{f}\right)$, with $E_{k}^{f}$ invertible, contains the finite zeros structure (i.e., the fixed finite poles of the inverse). Moreover, the matrices of the pair $\left(A_{k}^{\infty}, E_{k}^{\infty}\right)$ are in special staircase forms from which the infinity structure can be extracted by inspection (Note: The details of this extraction need to be still worked out). The maximum multiplicity of infinite zeros gives the minimum (or inherent) delay necessary to obtain a causal behavior for the inverse system. A remaining computational aspect is to obtain a standard system representation of the delay inverse by eliminating the infinite input decoupling zeros which arise after adding the delays at the outputs of the inverse system. An alternative possible approach along the lines of discussions in [11] is to determine the delay inverse by solving a model matching problem.

\section{B. Parameterization of All (1,2)-Inverses}

Following [12], all (1,2)-inverses of a matrix $W$ with coefficients in a field (e.g., the field of rational functions in $z$ ) can be parameterized as

$$
W^{+}=\left(X_{0}^{+}+N_{R} X\right)\left(Y_{0}^{+}+Y N_{L}\right)
$$

where $\widetilde{W}_{0}^{+}=X_{0}^{+} Y_{0}^{+}$is a full rank factorization of any particular $(1,2)$-inverse $\widetilde{W}_{0}^{+}$of $W, N_{R}$ and $N_{L}$ are basis matrices for the right and left nullspaces of $W$ (i.e., $W N_{R}=0$ and $N_{L} W=0$ ), and $X$ and $Y$ are arbitrary matrices with appropriate dimensions. By applying this parameterization to the generalized inverse of the lifted TFM $W_{k}(z)$ of a periodic system, we can obtain such a parameterization in terms of the $(1,2)$-inverse of the pencil $P_{k}(z)$ and possibly also in terms of periodic state-space realizations. Note that multiplications and additions of lifted TFMs correspond to series and parallel system couplings, respectively.

The main additional computational aspects to have such a parameterization is the computation of periodic realizations 
for the corresponding nullspaces. For the standard systems case, a reliable numerical algorithm has been proposed in [13] and recently extended to the periodic case [14].

\section{Computation of least order inverses}

A main application of the above parameterization is to determine (1,2)-inverses having least orders. Although this problem in the general (non full rank) case appears to be open even for standard systems, the least order aspect can be potentially addressed by our approach for computing particular (right or left) least order inverses by extending recently proposed techniques for standard systems (see for example [15]). The basic idea is to choose appropriate $F_{k}$ and/or $K_{k}$ to make a maximum number of spurious poles of the inverse system (21) unobservable or unreachable. For example, for a fixed $K_{k}$, choosing $F_{k}$ such that a maximum number of spurious poles corresponding to the pair $\left(A_{k}^{r}+\right.$ $B_{k}^{r} F_{k}^{1}, E_{k}^{r}$ ) becomes unreachable can be reformulated as a minimal order periodic dynamic cover design problem. The computational problem of determining minimal order dynamic covers for standard state space systems has been recently addressed in [16]. The proposed computational algorithm is essentially a modified staircase reachability form computation as that proposed in [17]. A similar algorithm for periodic systems has been proposed recently [18], and this algorithm could serve as basis to develop a similar cover design algorithm for periodic systems.

\section{NUMERICAL ISSUES}

The proposed computational approach to compute $(1,2)$ inverses relies on the reduction to the Kronecker-like form of a periodic pair by using a structure preserving numerically stable algorithm [11]. From the reduced pair, a $(1,2)$ inverse can be obtained simply by inspection, without any additional computation. Thus, the algorithm to compute a $(1,2)$-inverse is backward numerically stable. The overall computational complexity of this algorithm is at most $O\left(N q^{3}\right)$, where $q$ is an upper bound for the problem dimensions $\mu_{k}+p_{k}$ and $n_{k}+m_{k}$ [11]. Thus this is a satisfactory algorithm along the lines of requirements which we formulated in [7].

To obtain inverses with stable spurious poles, eigenvalue placement problems can be solved by using stabilization or pole assignment techniques for periodic descriptor systems with time-varying state and input dimensions. However, at present there is no reliable numerical algorithm developed for this computation. As an alternative approach, we can transform the underlying periodic descriptor systems into standard periodic systems and apply the algorithm of [19] which can address problems with time-varying dimensions

\section{CONCLUSIONS}

We proposed a numerically reliable method to compute $(1,2)$-generalized inverses of periodic systems. The proposed method is completely general, being applicable to both standard as well as descriptor periodic systems with time-varying state-, input-, and output -vector dimensions, having associated lifted TFMs of arbitrary rank. A particular emphasis has been put on reliably computing $(1,2)$-inverses, because of their relevance to many practical applications. The proposed approach provides flexibility to cope with various conditions on the spurious poles of the computed $(1,2)$-inverses. For instance, a stable $(1,2)$-inverse can be easily computed whenever exists. An important advantage of our computational approach to compute $(1,2)$-inverses is that periodic descriptor representations of inverses are obtained directly, without explicitly manipulating the lifted representations. In this way, computations with large dimensional sparse matrices or building of matrix products are completely avoided.

\section{ACKNOWLEDGMEnTS}

The work of the author has been performed in the framework of the Swedish Strategic Research Foundation Grant "Matrix Pencil Computations in Computer-Aided Control System Design: Theory, Algorithms and Software Tools".

\section{REFERENCES}

[1] M. Kono, "Invertibility of linear time-varying discrete-time systems," Systems \& Control Lett., vol. 1, pp. 148-153, 1981.

[2] A. M. Perdon, G. Conte, and S. Longhi, "Invertibility and inversion of linear periodic systems," Automatica, vol. 28, pp. 645-648, 1992.

[3] S. Longhi, A. M. Perdon, and G. Conte, "Geometric ans algebraic structure at infinity of discrete-time linear periodic systems," Lin. Alg. \& Appl., vol. 122/123/124, pp. 245-271, 1989.

[4] L. M. Silverman, "Inversion of multivariable linear systems," IEEE Trans. Automat. Control, vol. 14, pp. 270-276, 1969.

[5] A. Varga, "Computing generalized inverse systems using matrix pencil methods," Int. J. of Applied Mathematics and Computer Science, vol. 11, pp. 1055-1068, 2001.

[6] — - "Computation of minimal periodic realizations of transferfunction matrices," IEEE Trans. Automat. Control, vol. 46, pp. 146149, 2004.

[7] A. Varga and P. V. Dooren, "Computational methods for periodic systems - an overview," Proc. of IFAC Workshop on Periodic Control Systems, Como, Italy, 2001, pp. 171-176.

[8] O. M. Grasselli and S. Longhi, "Finite zero structure of linear periodic discrete-time systems," Int. J. Systems Sci., vol. 22, pp. 1785-1806, 1991.

[9] — "Zeros and poles of linear periodic discrete-time systems," Circuits, Systems and Signal Processing, vol. 7, pp. 361-380, 1988.

[10] G. Verghese, P. Van Dooren, and T. Kailath, "Properties of the system matrix of a generalized state-space system," Int. J. Control, vol. 30, pp. $235-243,1979$.

[11] A. Varga, "Computation of Kronecker-like forms of periodic matrix pairs," Proc. of MTNS'04, Leuven, Belgium, 2004.

[12] A. Ben Israel and T. N. E. Greville, Generalized Inverses: Theory and Applications. Wiley, New York, 1974.

[13] A. Varga, "On computing least order fault detectors using rational nullspace bases," Proc. of IFAC Symp. SAFEPROCESS'2003, Washington D.C., 2003.

[14] _ - "Design of fault detectors for periodic systems," Proc. of CDC'04, Paradise Island, Bahamas, 2004.

[15] — - "Computation of least order solutions of linear rational equations," Proc. of MTNS'04, Leuven, Belgium, 2004.

[16] _ "Reliable algorithms for computing minimal dynamic covers," Proc. of CDC'2003, Maui, Hawaii, 2003.

[17] _ , "Numerically stable algorithm for standard controllability form determination," Electron. Lett., vol. 17, pp. 74-75, 1981.

[18] _- "Computation of Kalman decompositions of periodic systems," European Journal of Control, vol. 10, pp. 1-8, 2004.

[19] S. Longhi and R. Zulli, "A note on robust pole assignment for periodic systems," IEEE Trans. Automat. Control, vol. 41, pp. $1493-$ 1497, 1996. 